\title{
Dexmedetomidine reduces propofol-induced apoptosis of neonatal rat hippocampal neurons via up-regulating Bcl-2 expression.
}

\author{
Li Yan, Qingfan Zeng*, Jing Wang, Jun Yong, Hongmei Xiao \\ Department of Anesthesiology, Baiyun Hospital Affiliated to Guizhou Medical University, PR China
}

\begin{abstract}
Background: The use of anesthetics propofol in certain pediatric age groups remains off-label, but it may cause neuronal apoptosis, long-term cognitive and neurological abnormalities during early postnatal period. Dexmedetomidine (Dex) can exert neuroprotective effects in rats. This study aimed to evaluate the effects of Dex and propofol on the apoptosis of neonatal rat hippocampal neurons.

Methods: Primary hippocampal neurons of neonatal rats were isolated and identified by immunofluorescence. Neurons were then divided into normal control, propofol $(12 \mu \mathrm{g} / \mathrm{ml}$ propofol $)$, and propofol+Dex group $(12 \mu \mathrm{g} / \mathrm{ml}$ propofol $+0.25 \mu \mathrm{g} / \mathrm{ml}$ Dex $)$. The cell morphology was detected by Hematoxylin-Eosin (HE) staining. The cell apoptosis was analysed by flow cytometry and Hoechst assay. The expression of Bcl-2 mRNA and protein was determined by quantitative reverse transcription PCR and Western blot.

Results: The apoptosis rate and proportion of Hoechst-positive cells in propofol group was significantly higher compared with control group $(\mathrm{p}=\mathbf{0 . 0 2 3}$ and $\mathbf{0 . 0 3 6}$, respectively). Propofol+Dex group exhibited significantly lower apoptosis and Hoechst-positive rate compared with propofol group ( $p=0.016$ and 0.028 , respectively). The expression of Bcl-2 mRNA and protein in propofol group was significantly decreased compared with control group ( $\mathrm{p}=0.012$ and 0.043 , respectively). The Bcl-2 mRNA and protein expression in propofol+Dex group was significantly higher than those in propofol group $(p=0.033$ and 0.026, respectively).

Conclusion: Propofol inhibited the growth and induced apoptosis of neonatal rat hippocampal neurons, whereas Dex suppressed the propofol-induced apoptosis via increasing the expression of Bcl-2. Our findings indicate that Dex in conjunction with propofol might reduce the propofol-induced neurotoxicity in pediatric patients who undergo general anesthetics, and therefore might provide an effective clinical measure against neural damages associated with general anesthetics.
\end{abstract}

Keywords: Propofol, Dexmedetomidine, Hippocampal neurons, Apoptosis, Bcl-2.

Accepted on December 21, 2017

\section{Introduction}

General anesthesia is widely used in a variety of surgeries. Propofol is an intravenous agent that is commonly used for the starting and maintenance of general anesthesia due to its pharmacological advantages over other anesthetic agents, such as rapid effect, short action, easy arousability and few side effects [1]. The efficacy and safety of propofol as an agent for the induction and maintenance of general anesthesia as well as sedation for children has been established in several clinical trials [2-4]. Propofol has received Food Drug and Administration (FDA) approval for induction of anesthesia only in children $\geq 3$ y of age and maintenance of anesthesia in children $\geq 2$ months of age [5]. The human brains develop rapidly from the embryonic period to 2 years after birth [6], and are extremely sensitive to anesthetic drugs. Therefore, the pediatric use of propofol in certain age groups remains offlabel [7], despite that the FDA issued a warning on the offlabel use of propofol in children in intensive care units [8].
Moreover, there have been discouraging literatures regarding its use in pediatric patients because propofol may cause neuronal apoptosis during the period of rapid development [9-11]. Propofol, administered during the early postnatal period, induces long-term cognitive and neurological abnormalities in neonatal rats [12].

Dexmedetomidine (Dex) is a highly selective agonist of $\alpha 2$ adrenergic receptors that can produce sedative, analgesic, sympatholytic effects without risks of respiratory depression [13]. When administered as an adjunct to general anesthesia, Dex not only minimizes the cardiovascular side-effects such as hypotension and bradycardia, but also reduces the required dosage of anesthetics [13-17]. Dexmedetomidine (Dex) can exert neuroprotective effects in rats [17]. Nevertheless, the benefit of Dex administration in conjunction with propofol for neurite growth and synapse development in neonates who underwent general anesthesia remains poorly studied. In this study, we isolated hippocampal neurons from neonatal 
Sprague-Dawley (SD) rats and investigated whether propofol could affect the growth of in vitro cultured hippocampal neurons. We further evaluated the effects of Dex on neuronal apoptosis when administrated in conjunction with propofol. Our study aimed to provide a theoretical basis for the clinical application of Dex as an adjunct to propofol for general anesthesia in pediatric patients, especially in neonates.

\section{Materials and Methods}

\section{Experimental animals and main reagents}

Clean grade neonatal SD rats (within $24 \mathrm{~h}$ after bone) weighting 4.5-6.0 g were purchased from the Nanchang University Animal Center. This study was approved by the Research Ethics Committee at the Nanchang University, and performed strictly in accordance with institutional and international guide for animal care. Neurobasal TM medium (Catalog number: 21103049), Fetal Bovine Serum (FBS), B-27TM supplement (50X), and serum free medium (Catalog number: 17504044) were purchased from GIBICO (Gaithersburg, MD, USA). L-glutamine (200 mM) and $\beta$-NGF were purchased from Solarbio (Shangha, China). Propofol was purchased from Fresenius Kabi Pharmaceutical Co., Ltd. (Graz, Austria). Dex was purchased from Jiangsu Hengrui Medicine Co. (Medicine registration number: H20130093, Lianyungang, China). TRIzon total RNA extraction kit, HiFiScript cDNA synthesis kit and UltraSYBR mixture was purchased from CWBIO (Beijing, China). Rabbit polyclonal anti-Bcl-2 antibody (ab32658), monoclonal anti-MAP-2 (microtubule associated protein 2) antibody (ab32454) and fluorescent-dye conjugated secondary antibody were purchased from Abcam (Cambridge, MA, USA).

\section{Isolation and culture of primary hippocampal neurons}

Neonatal SD rats were sacrificed by cervical dislocation. The brain was removed and put in per-chilled Hank's Balanced Salt Solution (HBSS). The pia mater and blood vessels were removed, and the bilateral hippocampus was isolated under an inverted microscope. The hippocampus was incubated in $0.125 \%$ trypsin at $37^{\circ} \mathrm{C}$ for $10-15 \mathrm{~min}$, rinsed twice with HBSS, and prepared into cell suspension in DMEM containing $10 \%$ FBS. The cell suspension was filtered through a sterile 70 $\mu \mathrm{m}$ filter, and seeded into DMEM supplemented with $10 \%$ FBS, 1\% sodium pyruvate and 1\% Gluta Max. After $4 \mathrm{~h}$, the medium was replaced with neurobasal medium containing $2 \%$ B27 and 1\% Gluta Max. Half of the medium was replaced every $3 \mathrm{~d}$.

\section{Immunofluorescence staining of primary hippocampal neurons}

The slide with primary hippocampal neuron monolayer was washed 3 times with PBS, fixed with 4\% paraformaldehyde for $15 \mathrm{~min}$, and treated with PBS containing $0.5 \%$ Triton X-100 (PBST). The slide was blocked with normal goat serum at room temperature for $30 \mathrm{~min}$, and incubated with primary antiMAP-2 antibody $(1: 200)$ overnight at $4^{\circ} \mathrm{C}$. The slide was rinsed thoroughly with PBST and incubated with fluorescentdye conjugated secondary antibodies at $37^{\circ} \mathrm{C}$ for $1 \mathrm{~h}$. The slide was further incubated with DAPI for $5 \mathrm{~min}$, treated with fluorescent quenching agent, and observed under a fluorescence microscope (Zeiss Axio Observer Z1).

\section{Grouping and treatment}

Cells were passed into 6-well plates and divided into 3 groups at $70 \%$ confluence: normal control, propofol $(12 \mu \mathrm{g} / \mathrm{ml}$ propofol), and propofol+Dex group $(12 \mu \mathrm{g} / \mathrm{ml}$ propofol +0.25 $\mu \mathrm{g} / \mathrm{ml}$ Dex). The plate was incubated at $37^{\circ} \mathrm{C}, 5 \% \mathrm{CO}_{2}$ in an incubator for $24 \mathrm{~h}$.

\section{Hematoxylin and eosin (HE) staining}

Cells in different groups were fixed with $4 \%$ paraformaldehyde for $15 \mathrm{~min}$, stained with hematoxylin for $3 \mathrm{~min}$, differentiated with hydrochloric acid for $15 \mathrm{~s}$, treated with $1 \%$ ammonia for $15 \mathrm{~s}$, and counterstained with eosin for $3 \mathrm{~min}$. The slide with cell monolayer was removed from each well, dehydrated in serial ethanol, sealed in neutral resin, and observed under an inverted microscope.

\section{Annexin V-FITC/PI apoptosis detection}

Cells at 70\% confluence in 6-well plates were incubated for 48 $\mathrm{h}$ in normal medium or medium with $12 \mu \mathrm{g} / \mathrm{ml}$ propofol or 12 $\mu \mathrm{g} / \mathrm{ml}$ propofol $+0.25 \mu \mathrm{g} / \mathrm{ml}$ Dex. Cells were collected and stained using Annexin V-FITC/PI apoptosis detection kit according to the manufacturer's instructions. Cell apoptosis was detected using a FACSCalibur flow cytometer (BD Biosciences, San Jose, CA, USA) within $1 \mathrm{~h}$.

\section{Hoechst apoptosis detection}

Cells were seeded in 6-well plates with sterile coverslip and incubated until $70 \%$ confluence. Cells were then incubated for $48 \mathrm{~h}$ in regular medium or medium with $12 \mu \mathrm{g} / \mathrm{ml}$ propofol or $12 \mu \mathrm{g} / \mathrm{ml}$ propofol $+0.25 \mu \mathrm{g} / \mathrm{ml}$ Dex. The coverslip was removed from the plate, fixed with $4 \%$ paraformaldehyde for $10 \mathrm{~min}$, rinsed with PBS, and stained with $0.5 \mathrm{ml}$ Hoechst 33258 for $5 \mathrm{~min}$. The coverslip was then put on a slide with fluorescent quenching agent, and observed under a fluorescence microscope (excitation wavelength: $350 \mathrm{~nm}$ and emission wavelength: $460 \mathrm{~nm}$ ).

\section{Quantitative reverse transcription PCR (qRT-PCR)}

Total RNA was extracted from cells in different groups using TRIzon reagent. The quality of total RNA was assessed by measuring the A260/280 ratio. Reverse transcription was performed using the HiFiScript cDNA synthesis kit. The qRTPCR reaction was performed on a CFX96 real-time PCR system (Bio-Rad, Hercules, CA, USA) using UltraSYBR mixture. The reaction mixture was prepared: cDNA $1 \mu$, forward primer $1 \mu \mathrm{l}$, reverse primer $1 \mu \mathrm{l}$, 2X UltraSYBR mix $12.5 \mu \mathrm{l}$ and $\mathrm{H}_{2} \mathrm{O} 9.5 \mu \mathrm{l}$. The reaction conditions were as 
follows: $95^{\circ} \mathrm{C} 10 \mathrm{~min}$ followed by 40 cycles of $95^{\circ} \mathrm{C} 10 \mathrm{~s}$, $56.5^{\circ} \mathrm{C} 30 \mathrm{~s}$, and $72^{\circ} \mathrm{C} 30 \mathrm{~s}$. The melting curve analysis was as follows: $95^{\circ} \mathrm{C} 15 \mathrm{~s}, 56.5^{\circ} \mathrm{C} 1 \mathrm{~min}, 95^{\circ} \mathrm{C} 15 \mathrm{~s}, 56.5^{\circ} \mathrm{C} 15 \mathrm{~s}$, $56.5^{\circ} \mathrm{C} 15 \mathrm{~s}$, and $95^{\circ} \mathrm{C} 0.5 \mathrm{~s}$. The primers for $\mathrm{Bcl}-2$ and GAPDH were synthesized by Genscript (Nanjing, China): BCL-2 F: 5'-CAGGCTGGAAGGAGAAGATGC-3', BCL-2 R: 5'-AGAGGGGCTACGAGTGGGATA-3', GAPDH F: 5'GCAAGTTCAACGGCACAG-3', and GAPDH R: 5'CGCCAGTAGACTCCACGAC-3'. The experiment was performed in triplicate and the expression level was calculated using the $2^{-\Delta \mathrm{Ct}}$ method. The GAPDH was used as the internal control.

\section{Western blot}

Cells in different groups were treated in lysis buffer (Beyotime, Shanghai, China) for $30 \mathrm{~min}$. Total protein was obtained by centrifugation at $10000 \mathrm{rpm} / \mathrm{min}, 4^{\circ} \mathrm{C}$ for $10 \mathrm{~min}$, and quantified using BCA Protein Assay Kit (Beyotime). Equal aliquotes $(50 \mathrm{mg})$ of protein were then separated by $10 \%$ SDSPAGE and transferred to polyvinylidene difluoride membranes. Membranes were blocked with 5\% skim milk for $30 \mathrm{~min}$, and successively incubated with rabbit anit-Bcl-2 antibody overnight at $4{ }^{\circ} \mathrm{C}$ and with HRP-conjugated secondary antibody at room temperature for $30 \mathrm{~min}$. The immunoreactivity was detected using the ChemiDocTM XRS gel imaging system (Bio-Rad, USA). The relative expression of proteins was quantified using Quantity One 4.6.1 software (Bio-Rad) with GAPDH as the internal reference.

\section{Transmission electron microscopy (TEM)}

Cells in different groups were fixed with $2.5 \%(\mathrm{w} / \mathrm{v})$ glutaraldehyde solution in $0.1 \mathrm{M}$ cold phosphate buffer for $2 \mathrm{~h}$, treated with $1 \%$ osmium acid for $2 \mathrm{~h}$, and incubated successively in acetone + embedding solution $(2: 1)$ at room temperature for $3 \mathrm{~h}$, acetone+embedding solution (1:2) overnight at room temperature, and embedding solution at $37^{\circ} \mathrm{C}$ for $2 \mathrm{~h}$. The block was cut into $70 \mathrm{~nm}$ sections using an Leica EM UC7 ultra-thin slicer, stained with 3\% uranyl acetate and lead citrate, and observed using a JEOL JEM-1230 electron microscope (Bio-Rad) at $80 \mathrm{kV}$.

\section{Statistical analysis}

All data were represented as mean \pm Standard Error of the Mean (SEM) and analysed using SPSS 17.0 software (IBM SPSS., Chicago, TX, USA). Differences among groups were compared by one-way ANOVA followed by post-hoc LSD tests. $\mathrm{p}$ values smaller than 0.05 was considered statistically significant.

\section{Results}

\section{Dex reversed the inhibitory effect of propofol on neuronal growth}

The expression of MAP-2, a neuron-specific marker, was detected by fluorescence staining. As shown in Figure 1, the in vitro cultured cells were positive for MAP-2, indicating the successful isolation and cultivation of primary hippocampal neurons. Further, the hippocampal neurons were allocated to control, propofol and propofol+Dex groups, and the morphology of cells in different groups were compared by HE staining. As shown in Figure 2, a large number of pyramidal cells with abundant cytoplasm and big nucleus were observed in the control group. The cells in propofol group were clearly smaller compared with control group. In contrast, the cell morphology in propofol+Dex group was similar to that in control group, suggesting that propofol had inhibited the development of hippocampal neurons, whereas Dex reversed such effect.

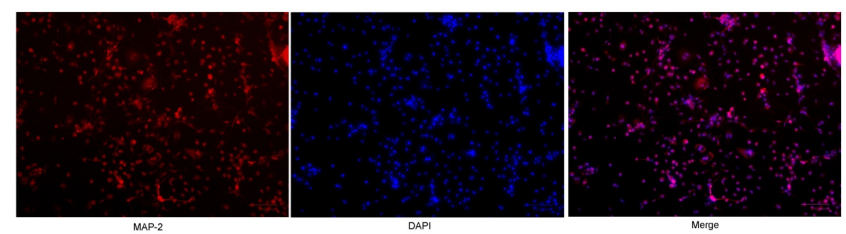

Figure 1. Representative fluorescence microscopic images of primary hippocampal neurons. Primary hippocampal neurons were isolated from neonatal rats, cultured in neurobasal medium containing $2 \%$ B27 and 1\% Gluta Max for 48 h, subjected to immunofluorescence staining (200X). Immunofluorescence of neuron-specific marker MAP-2 confirms the identity of cultured primary hippocampal neurons.
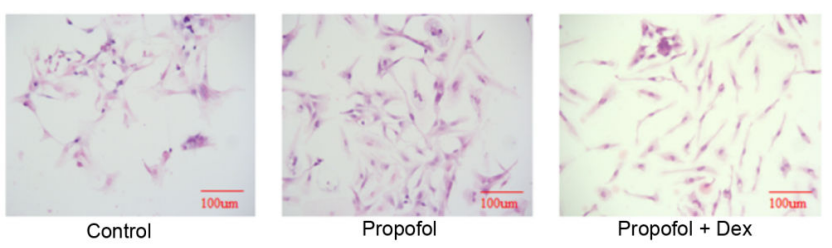

Figure 2. Comparison of the morphology of hippocampal neurons in different groups by HE staining. Hippocampal neurons were incubated in normal medium or medium with $12 \mu \mathrm{g} / \mathrm{ml}$ propofol or 12 $\mu \mathrm{g} / \mathrm{ml}$ propofol $+0.25 \mu \mathrm{g} / \mathrm{ml}$ Dex for $24 \mathrm{~h}$, and subjected to $H E$ staining (200X).

\section{Dex reduced the stimulatory effect of propofol on neuronal apoptosis}

The morphology of cells in different groups was observed under TEM. As shown in Figure 3, cells in the control group had large nucleus with irregular nuclear membrane and little heterochromatic chromatin. In contrast, the cells in propofol group were markedly smaller. Increased heterochromatic chromatin and broken cell nucleus were observed. The number of synapses of hippocampal neurons was obviously lower than that in control group. The cells in propofol+Dex group exhibited large, round nucleus with smooth nuclear membrane, clear nucleolus, and little heterochromatic chromatin, and had clearly more synapses compared with control group. The cell apoptosis in each group was compared by both annexin VFITC/PI staining and Hoechst staining. It was found that the apoptosis rate and proportion of Hoechst-positive cells in propofol group was significantly higher compared with the control group ( $\mathrm{p}=0.023$ and 0.036 , respectively). The propofol 
+Dex group exhibited significantly lower apoptosis rate and Hoechst-positive rate than those in propofol group $(\mathrm{p}=0.016$ and 0.028, respectively, Figure 4). All together, these observations suggested that propofol had stimulated the apoptosis of hippocampal neurons, and Dex exerted protective effect against such effect and reduced neuronal apoptosis.
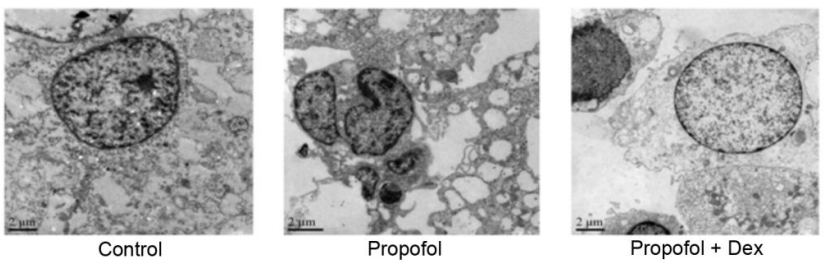

Figure 3. Transmission microelectroscope images showing the morphology of hippocampal neurons in different groups. Hippocampal neurons were incubated in normal medium or medium with $12 \mu \mathrm{g} / \mathrm{ml}$ propofol or $12 \mu \mathrm{g} / \mathrm{ml}$ propofol $+0.25 \mu \mathrm{g} / \mathrm{ml}$ Dex for 48 $h$, and observed under a transmission microelectroscope.
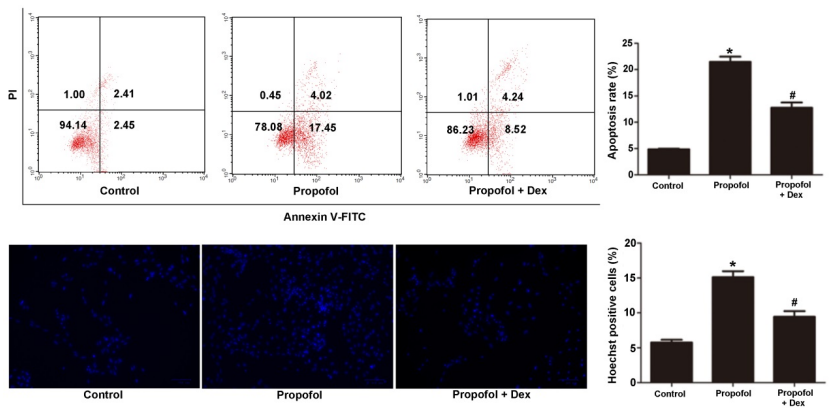

Figure 4. Dex reduced the stimulatory effect of propofol on neuronal apoptosis. Hippocampal neurons were incubated in normal medium or medium with $12 \mu \mathrm{g} / \mathrm{ml}$ propofol or $12 \mu \mathrm{g} / \mathrm{ml}$ propofol $+0.25 \mu \mathrm{g} / \mathrm{ml}$ Dex for $48 \mathrm{~h}$, and subjected to Annexin V-FITC/PI staining /flow cytometry (A) and Hoechst staining (B). Hippocampal neuronal apoptosis in different groups was compared. ${ }^{*} P<0.05$, compared with control group; ${ }^{*} p<0.05$, compared with propofol group.
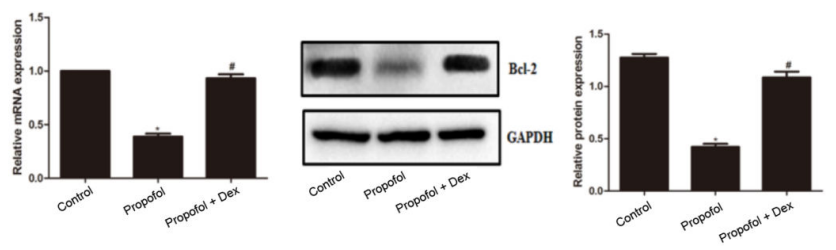

Figure 5. Dex reversed the inhibitory effect of propofol on Bcl-2 expression. Hippocampal neurons were incubated in normal medium or medium with $12 \mu \mathrm{g} / \mathrm{ml}$ propofol or $12 \mu \mathrm{g} / \mathrm{ml}$ propofol $+0.25 \mu \mathrm{g} / \mathrm{ml}$ Dex for $48 \mathrm{~h}$, and Bcl-2 expression was analysed by $q R T-P C R$ (a) and Western blot (b). ${ }^{*} P<0.05$, compared with control group; ${ }^{*} p<0.05$, compared with propofol group.

\section{Dex reversed the inhibitory effect of propofol on Bcl-2 expression}

The expression of Bcl-2 in each group was determined by qRTPCR and Western blot analyses. As shown in Figure 5, the expression of Bcl-2 mRNA and protein in propofol group was significantly decreased compared with control group $(p=0.012$ and 0.043 , respectively), suggesting that propofol had reduced the Bcl-2 expression in hippocampal neurons. The Bcl-2 mRNA and protein expression in propofol+Dex group was significantly higher than those in propofol group $(\mathrm{p}=0.033$ and 0.026 , respectively), and was comparable to those in control group (both $\mathrm{p}>0.05$ ). These results revealed that Dex had reversed the inhibitory effect of propofol on Bcl-2 expression.

\section{Discussion}

In vitro cultured primary neurons have been used as an important experimental model for a wide range of neural researches [18]. The hippocampus is one of the brain areas that are sensitive to anesthetics during general anesthesia, which may lead to central nervous damages. Therefore, we used primary hippocampal neurons from neonatal SD rats as an in vitro model to investigate the neurotoxicity of propofol and the protective effect of Dex against such neurotoxicity.

MAP-2 is a neuron-specific cytoskeletal protein that stabilizes microtubules growth by crosslinking with intermediate filaments and other microtubules during neuronal development. MAP2 is expressed mainly in neuronal axons and dendritic extensions $[19,20]$, and thus is commonly used as a marker of neuronal phenotype [21]. In this study, we successfully isolated and cultured primary hippocampal neurons from neonatal SD rats as indicated by the strong MAP-2 immunoreactivity.

The neurotoxicity of propofol have been reported in several types of neurons, including human stem cell-derived neurons, rat prefrontal cortical neurons and hypoglossal motoneurons, as well as rodent developing hippocampal astrocytes [22-25]. Consistently, we found that propofol had markedly inhibited the growth and stimulated the apoptosis of rat hippocampal neurons. Bcl-2 is a key member of the large Bcl-2 family of regulator proteins that either induce (pro-apoptotic) or inhibit (anti-apoptotic) apoptosis [26]. BCL-2 is an important antiapoptotic protein localized to the outer membrane of mitochondria, where it inhibits the activities of pro-apoptotic proteins and promotes cellular survival [27]. Propofol may promote the hippocampal neuronal apoptosis via up-regulating Bcl-2 expression and down-regulating protein kinase caspase-3 expression [28]. Consistently, our study also found that propofol reduced the expression of Bcl-2 mRNA and protein in hippocampal neurons when compared with control group without any treatment. Moreover, a decreased number of synapses of hippocampal neurons is known to be associated with damages in the hippocampal nerve, leading to impaired cognitive competence and memory, and aggravated anxiety of rats [29]. In this study, TEM findings revealed that propofoltreated hippocampal neurons had obviously less synapses compared with control group. All together, these results suggested that propofol had exerted neurotoxicity on in vitro cultured rat primary hippocampal neurons.

Dex acts on the central nervous system through inhibiting the activity of sympathetic nerve, which produces a variety of effects such as anti-anxiety, sedation, analgesia, etc. It also 
improves cognitive dysfunction, attenuates microcirculatory derangements, and reduces delirium after surgeries [30-32]. Most importantly, Dex can exert neuroprotective effects by stimulating the expression of Brain-Derived Neurotrophic Factor (BDNF) [17,33]. Intraperitoneally injected Dex can attenuate the isoflurane-induced toxicity to the brain of newborn rats via promoting Bcl-2 expression, and thus protect neural damages [34]. We detected a significantly lower hippocampal neural apoptosis rate and Hoechst-positive rate in propofol+Dex group when compared with propofol group. We also found that propofol+Dex group had a significantly higher Bcl-2 mRNA and protein expression than those in propofol group, suggesting that Dex effectively inhibited the stimulatory effect of propofol on neuronal apoptosis. Furthermore, TEM result demonstrated that propofol+Dex group had clearly more synapses of hippocampal neurons compared with control group, indicating that Dex attenuated the propofol-induced neuronal damages.

The current study is largely limited by the lack of animal studies. Our findings need to be verified using animal models. Furthermore, we only performed preliminary mechanistic study. The investigation of molecular mechanism will be our next research focus, such as whether BDNF or inflammatory cytokines are involved in the protective effect of Dex against propofol-induced neurotoxicity.

\section{Conclusion}

In summary, our study found that propofol inhibited the growth and induced apoptosis of neonatal rat hippocampal neurons, whereas Dex suppressed the propofol-induced apoptosis via increasing the expression of Bcl-2. Our findings suggest that Dex in conjunction with propofol might reduce the propofolinduced neurotoxicity in pediatric patients who undergo general anesthetics, and therefore might provide an effective clinical measure against neural damages associated with general anesthetics.

\section{Ethics Statement}

The animal study in this work was approved by the Animal Ethics Committee at the Jiangxi Provincial People's Hospital.

\section{Conflict of Interests}

None

\section{Acknowledgments}

This study was supported by the project of Guizhou Bureau of Science and Technology (project No.: LH (2015) 7389).

\section{References}

1. Chidambaran V, Costandi A, DMello A. Propofol: a review of its role in pediatric anesthesia and sedation. CNS Drugs 2015; 29: 543-563.

2. Reed MD, Yamashita TS, Marx CM, Myers CM, Blumer JL. A pharmacokinetically based propofol dosing strategy for sedation of the critically ill, mechanically ventilated pediatric patient. Crit Care Med 1996; 24: 1473-1481.

3. Angelini G, Ketzler JT, Coursin DB. Use of propofol and other nonbenzodiazepine sedatives in the intensive care unit. Crit Care Clin 2001; 17: 863-880.

4. Kulling D, Rothenbuhler R, Inauen W. Safety of nonanesthetist sedation with propofol for outpatient colonoscopy and esophagogastroduodenoscopy. Endoscopy 2003; 35: 679-682.

5. Orange Book: approved drug products with therapeutic equivalence evaluations 2011. US Food and Drug Administration. http: //www.accessdata.fda.gov/scripts/ cder/ob/docs/temptn.cfm. Accessed September 26, 2017.

6. Knickmeyer RC, Gouttard S, Kang C, Evans D, Wilber K, Smith JK, Hamer RM, Lin W, Gerig G, Gilmore JH. A structural MRI study of human brain development from birth to 2 years. J Neurosci 2008; 28: 12176-12182.

7. Smith MC, Williamson J, Yaster M, Boyd GJ, Heitmiller ES. Off-label use of medications in children undergoing sedation and anesthesia. Anesth Analg 2012; 115: 1148-1154.

8. Canadian Medical Association. FDA issues warning on propofol (Diprivan). CMAJ 2001; 164: 1608.

9. Toman H, Erkilinc A, Kocak T, Guzelmeric F, Savluk OF, Dogukan M, Acar G. Sedation for transesophageal echocardiography: comparison of propofol, midazolam and midazolam-alfentanil combination. Med Glas (Zenica) 2016; 13: 18-24.

10. Maldifassi MC, Baur R, Sigel E. Functional sites involved in modulation of the GABAA receptor channel by the intravenous anesthetics propofol, etomidate and pentobarbital. Neuropharmacology 2016; 105: 207-214.

11. Mir AH, Shah NF, Din MU, Langoo SA, Reshi FA. Effectiveness of sodium thiopentone, propofol, and etomidate as an ideal intravenous anesthetic agent for modified electroconvulsive therapy. Saudi J Anaesth 2017; 11: 26-31.

12. $\mathrm{Xu} C$, Seubert CN, Gravenstein N, Martynyuk AE. Propofol, but not etomidate, increases corticosterone levels and induces long-term alteration in hippocampal synaptic activity in neonatal rats. Neurosci Lett 2016; 618: 1-5.

13. Wu X, Hang LH, Wang H, Shao DH, Xu YG, Cui W, Chen Z. Intranasally administered adjunctive dexmedetomidine reduces perioperative anesthetic requirements in general anesthesia. Yonsei Med J 2016; 57: 998-1005.

14. Jin S, Liang DD, Chen C, Zhang M, Wang J. Dexmedetomidine prevent postoperative nausea and vomiting on patients during general anesthesia: A PRISMA-compliant meta-analysis of randomized controlled trials. Medicine (Baltimore) 2017; 96: 5770.

15. Amorim MA, Govêia CS, Magalhães E, Ladeira LC, Moreira LG, Miranda DB. Effect of dexmedetomidine in children undergoing general anesthesia with sevoflurane: a meta-analysis. Braz J Anesthesiol 2017; 67: 193-198.

16. Wu HH, Wang HT, Jin JJ, Cui GB, Zhou KC, Chen Y, Chen GZ, Dong YL, Wang W. Does dexmedetomidine as a 
neuraxial adjuvant facilitate better anesthesia and analgesia? A systematic review and meta-analysis. PLoS One 2014; 9: 93114.

17. Hwang L, Choi IY, Kim SE, Ko IG, Shin MS, Kim CJ, Kim SH, Jin JJ, Chung JY, Yi JW. Dexmedetomidine ameliorates intracerebral hemorrhage-induced memory impairment by inhibiting apoptosis and enhancing brainderived neurotrophic factor expression in the rat hippocampus. Int J Mol Med 2013; 31: 1047-1056.

18. Facci L, Skaper SD. Culture of rodent cortical and hippocampal neurons. Methods Mol Biol 2012; 846: 49-56.

19. Harada A, Teng J, Takei Y, Oguchi K, Hirokawa N. MAP2 is required for dendrite elongation, PKA anchoring in dendrites, and proper PKA signal transduction. J Cell Biol 2002; 158: 541-549.

20. Dehmelt L, Halpain S. The MAP2/Tau family of microtubule-associated proteins. Genome Biol 2005; 6: 204.

21. Liu Y, Saad RS, Shen SS, Silverman JF. Diagnostic value of microtubule-associated protein-2 (MAP-2) for neuroendocrine neoplasms. Adv Anat Pathol 2003; 10: 101-106.

22. Deng X, Chen B, Wang B, Zhang J, Liu H. TNF-a mediates the intrinsic and extrinsic pathway in propofol-induced neuronal apoptosis via PI3K/Akt signaling pathway in rat prefrontal cortical neurons. Neurotox Res 2017; 32: 409-419.

23. Monni L, Ghezzi F, Corsini S, Nistri A. Neurotoxicity of propofol on rat hypoglossal motoneurons in vitro. Neurosci Lett 2017; 655: 95-100.

24. Twaroski DM, Yan Y, Olson JM, Bosnjak ZJ, Bai X. Down-regulation of microRNA-21 is involved in the propofol-induced neurotoxicity observed in human stem cell-derived neurons. Anesthesiology 2014; 121: 786-800.

25. Sun WC, Liang ZD, Pei L. Propofol-induced rno-miR-665 targets BCL2L1 and influences apoptosis in rodent developing hippocampal astrocytes. Neurotoxicology 2015; 51: 87-95.

26. Kale J, Osterlund EJ, Andrews DW. BCL-2 family proteins: changing partners in the dance towards death. Cell Death Differ 2018; 25: 65-80.
27. Opferman JT, Kothari A. Anti-apoptotic BCL-2 family members in development. Cell Death Differ 2018; 25: 37-45.

28. Zhong Y, Liang Y, Chen J. Propofol inhibits proliferation and induces neuroapoptosis of hippocampal neurons in vitro via downregulation of $\mathrm{NF}-\kappa \mathrm{B}$ p65 and $\mathrm{Bcl}-2$ and upregulation of caspase-3. Cell Biochem Funct 2014; 32: 720-729.

29. Mayford M, Siegelbaum SA, Kandel ER. Synapses and memory storage. Cold Spring Harb Perspect Biol 2012; 4.

30. Qian XL, Zhang W, Liu MZ, Zhou YB, Zhang JM, Han L, Peng YM, Jiang JH, Wang QD. Dexmedetomidine improves early postoperative cognitive dysfunction in aged mice. Eur J Pharmacol 2015; 746: 206-212.

31. Miranda ML, Balarini MM, Bouskela E. Dexmedetomidine attenuates the microcirculatory derangements evoked by experimental sepsis. Anesthesiology 2015; 122: 619-630.

32. Djaiani G, Silverton N, Fedorko L, Carroll J, Styra R, Rao V, Katznelson R. Dexmedetomidine versus propofol sedation reduces delirium after cardiac surgery: a randomized controlled trial. Anesthesiology 2016; 124: 362-368.

33. Degos V, Charpentier TL, Chhor V, Brissaud O, Lebon S, Schwendimann L, Bednareck N, Passemard S, Mantz J, Gressens P. Neuroprotective effects of dexmedetomidine against glutamate agonist-induced neuronal cell death are related to increased astrocyte brain-derived neurotrophic factor expression. Anesthesiology 2013; 118: 1123-1132.

34. Cheng XY, Gu XY, Gao Q, Zong QF, Li XH, Zhang Y. Effects of dexmedetomidine postconditioning on myocardial ischemia and the role of the PI3K/Aktdependent signaling pathway in reperfusion injury. Mol Med Rep 2016; 14: 797-803.

\section{*Correspondence to}

Qingfan Zeng

Department of Anesthesiology

Baiyun Hospital Affiliated to Guizhou Medical University

PR China 